\title{
Situación taxonómica de las especies del género Polylepis. Implicancias para los estudios ecológicos, la conservación y la restauración de sus bosques
}

\author{
María C. Segovia-Salcedo ${ }^{1,0}$; Alejandra Domic ${ }^{2}$; Tatiana E. Boza ${ }^{3}$ \& Michael \\ KESSLER $^{3}$ \\ ${ }^{1}$ Departamento de Ciencias de la Vida y Agricultura. Universidad de las Fuerzas Armadas ESPE. Sangolquí. Ecuador. ${ }^{2}$ Centro \\ de Estudios Avanzados en Zonas Áridas, Santiago, Región de Coquimbo, Chile. ${ }^{3}$ Department of Systematic and Evolutionary \\ Botany, University of Zurich, Switzerland.
}

\begin{abstract}
Resumen. Según los diversos tratamientos taxonómicos, el número de especies del género Polylepis oscila entre 33 y 28 especies. Sin embargo, hasta el momento no se pudieron delimitar claramente las especies de este género. Es difícil definir sus orígenes evolutivos y conocer los procesos de especiación. Esta complejidad obedecería a distintos procesos que actúan de forma independiente y sinérgica dependiendo del grupo de especies: diferenciación relativamente reciente, poliploidía, hibridación y el aparente rol del ser humano en su distribución. En este artículo realizamos una revisión de la situación taxonómica actual del género Polylepis. Proponemos 28 especies e incluimos 2 nuevas especies descriptas para Perú y una especie (P. tomentella) con tres subespecies. Además, discutimos los vacíos en el conocimiento y proponemos estrategias de investigación que permitan mejorar la delimitación de las especies. A fin de proveer una estructura taxonómica dentro de los estudios y para que los datos obtenidos constituyan un aporte a los procesos de conservación de estos bosques, taxónomos y conservacionistas debemos trabajar en conjunto para delimitar las unidades de conservación que eviten ambigüedades y para, de esta manera, definir estrategias claras.
\end{abstract}

[Palabras clave: delimitación de especies, Rosáceas, taxonomía compleja]

\begin{abstract}
Taxonomic review of the genus Polylepis. Implications for ecological studies, conservation and restoration. The number of species in the genus Polylepis has changed, according to the systematic treatments, from 33 to 28; however, until now there is not a clear species delimitation. It has been complicated to define evolutionary origins and understand speciation. This complexity may be the result of different processes acting independently or interacting: recent radiation, poliploidy, hibridization and the human intervention in its distribution, depending of the species group. In this article, we revised the taxonomic status of the genus Polylepis. Twenty-eight species are proposed, with the inclusion of two new species for Perú and one species ( $P$. tomentella) with three subespecies. In addition, gaps of knowledge are analyzed and strategies of research are proposed to improve species delimitation. Incorporating a taxonomic structure could generate new insights to conservation, restoration and management projects; taxonomists and conservationist need to work together to define conservation units to avoid ambiguities and define new strategies.
\end{abstract}

[Keywords: species delimitation, Rosaceae, complex taxonomy]

\section{INTRODUCCIÓN}

Polylepis es un género que incluye aproximadamente 28 especies de árboles y arbustos distribuidos a lo largo de la Cordillera de los Andes, desde Venezuela hasta Chile y la Argentina (Simpson 1979). El género se caracteriza por una distribución dispersa en forma de fragmentos boscosos que crecen en laderas montañosas, quebradas rocosas $\mathrm{y}$ riachuelos. Se presumen que el género evolucionó durante el levantamiento de los Andes durante el Plio-Pleistoceno (Simpson 1986; Eriksson et al. 2003; Kerr 2003). Dentro de Polylepis existe un gradiente morfológico y ecológico en el que las especies con individuos altos, hojas delgadas y múltiplesinflorescencias habitan el límite inferior de los bosques montanos y las elevaciones bajas, mientras que aquellas con individuos pequeños, hojas coriáceas y pequeñas inflorescencias crecen en hábitats semiáridos ubicados en sitios elevados (Simpson 1979, 1986; Kessler 1995; Kerr 2003). A pesar de que el género no es uno de los más diversos de la flora altoandina, constituye un ejemplo interesante de cómo un género vegetal evolucionó rápidamente y colonizó diversos nichos durante la formación de la Cordillera de los Andes (Schmidt-Lebuhn et al. 2010).

Editor asociado: Daniel Renison 
En comparación con otros países andinos, los que poseen la mayor cantidad de especies son Perú, Bolivia y Ecuador (Simpson 1979; Kessler and Schmidt-Lebuhn 2006). Hasta la fecha, existen varios estudios taxonómicos sobre Polylepis que delimitaron las especies sobre la base de caracteres morfológicos (Ruiz and Pavón 1794; Bitter 1911; Simpson 1979; Kessler 1995). Más recientemente, otros autores lo hicieron a partir de marcadores moleculares (Kessler and Schmidt-Lebuhn 2006). Sin embargo, todavía es incierto el número exacto de especies existentes $\mathrm{y}$, sobre todo, existen vacíos de información acerca de los procesos de especiación que dieron origen al género y a su posterior radiación. La problemática taxonomía de Polylepis se refleja en la discrepancia entre las revisiones taxonómicas propuestas (Bitter 1911; Simpson 1979; Kessler 1995; Kessler and SchmidtLebuhn 2006), basada en la dificultad para delimitar algunas especies por la plasticidad fenotípica, la hibridación y la falta de diferenciación genética (Kerr 2003).

El género Polylepis es un componente clave de la flora andina. Por ello, entender el origen biogeográfico también puede ayudar a comprender la historia biogeográfica de la flora de la región. Al mismo tiempo, la delimitación taxonómica y la caracterización de la diversidad de especies tienen implicancias clave para su conservación. Actualmente, muchas especies se encuentran amenazadas. Se estima que Polylepis poseía una distribución más extensa en el pasado y que las actividades humanas (e.g., tala, quema, pastoreo y agricultura) y el cambio climático produjeron la pérdida y la degradación de los bosques. En el presente artículo realizamos una revisión bibliográfica con el fin de sistematizar los tratamientos taxonómicos y sistemáticos del género Polylepis, y para identificar áreas prioritarias de investigación que permitan mejorar la delimitación de las especies. Además, incluimos un análisis sobre la importancia de realizar estudios biológicos y ecológicos.

\section{Materiales y Métodos}

Se realizó una revisión bibliográfica de los tratamientos taxonómicos y de los artículos científicos existentes acerca del género Polylepis con la finalidad de definir el número de especies descritas hasta el momento $y$, al mismo tiempo, para detectar los vacíos de conocimiento en relación a este género. Para obtener la mayor cantidad de información se utilizaron las bases de datos de Science Direct y el buscador Google Académico con las palabras clave Polylepis y Andes. Además, cada uno de los autores contribuyó con su experiencia en el tema basado en su trabajo de campo y análisis de material de herbario.

\section{Resultados}

Revisión de estudios taxonómicos y sistemáticos del género Polylepis

El género Polylepis Ruiz and Pav. y su primera especie, $P$. racemosa, fueron descritos en 1798 por Ruiz y Pavón. Durante el siglo XIX, varios autores describieron 15 formas adicionales de Polylepis, algunas a nivel de especie y otras a nivel de subespecie o variedad (Hieronymus 1895; Kunth 1898; Pilger 1906), con otras dos especies descritas en la primera década del siglo XX (Kessler and Schmidth-Lebunh 2006).

El primer trabajo taxonómico que trata el género completo fue publicado en 1911 por Bitter, quien reconoció todas las especies descritas hasta entonces (33 especies), elevó dos subespecies y una variedad a nivel de especie y describió 13 nuevas especies, 9 subespecies y 18 variedades. Desafortunadamente, Bitter (1911) no realizó muchas colectas botánicas a campo y, en consecuencia, no consideró la variabilidad morfológica intraespecífica de las especies en el estudio taxonómico. Como resultado de esto, él utilizó un concepto taxonómico tipológico en el que cada ejemplar con ligeras diferencias fue asignado con nombre diferente. Por lo tanto, la taxonomía utilizada por Bitter (1911) fue poco clara y difícil de utilizar. Actualmente, la mayoría de los taxones descritos por Bitter no son aceptados.

En los años subsiguientes, Bitter (1913) describió una variedad adicional, mientras que otros autores nombraron cinco especies más (Benoist 1934; Macbride 1934; Cuatrecasas 1941, 1942). En 1979, Simpson revisó la taxonomía del género $y$, en base a trabajo de campo, obtuvo una buena idea de la variabilidad natural de las especies, reconociendo que muchos de los nombres usados en el género se aplicaban a la misma especie. Como resultado de esto, ella redujo el número de especies reconocidas de 38 a 14 y describió una especie adicional, resultando en 15 especies. La autora no aceptó taxones 
infraespecíficos. Confrontada con un número enorme de nombres sin base biológica, es fácil de entender que Simpson (1979) tuviera una tendencia a reconocer especies ampliamente definidas, utilizando un concepto biológico de especie. Como resultado, algunas de las especies que ella reconoció fueron muy variables $y$, en publicaciones subsiguientes, ella misma manifestó que algunas de sus "especies" serían mejor tratadas como "grupos de especies" (Simpson 1986), aunque con excepción de $P$. tarapacana Phil. como especie distinta de P. tomentella Wedd., ella no separó estos grupos en especies individuales.

Desde entonces, se realizaron trabajos taxonómicos en Polylepis mayormente a nivel nacional. Romoleroux (1985) trató a las especies ecuatorianas según el concepto de Simpson (1979), pero reconoció a P. microphylla (Wedd.) Bitter como especie independiente de $P$. weberbaueri Pilg. Kessler (1995) revisó las especies bolivianas a partir de un concepto de especie similar al de Simpson (1979), pero reconociendo variaciones geográficas a nivel de subespecies; así, describió una especie nueva. Además, revalidó la especie $P$. rugulosa Bitter para Chile y Perú (Kessler 1995). Estos conceptos taxonómicos fueron adoptados por Fjeldså y Kessler (1996). Sin embargo, en los años subsiguientes, en base a una revisión de los caracteres morfológicos y al empleo de marcadores moleculares, Kessler y SchmidtLebuhn (2006) modificaron el concepto taxonómico y reconocieron como especies a formas geográficas y morfológicamente separadas $(P$. flavipila, $P$. incarum, $P$. lanata y P. subtusalbida), que antes habían sido tratadas como subespecies; también describieron una especie nueva ( $P$. pacensis) y re-instauraron a una especie adicional ( $P$. triacontandra). Como resultado de esta última revisión, se reconocieron en total 26 especies de Polylepis.

La revisión taxonómica más reciente la llevaron a cabo Mendoza y Cano (2011). Ellos centraron el estudio en Perú, reconocieron 19 especies para ese país e incluyeron una especie descrita en 2005, P. canoi (Mendoza 2005), y varios nuevos registros de especies antes sólo conocidas en Ecuador y Bolivia. En general, el concepto de especie aplicado es el mismo que el que usaran Kessler y Schmidt-Lebuhn (2006); sin embargo, la delimitación taxonómica de las especies difiere parcialmente, de manera que los nombres no son utilizados de manera idéntica, como en el caso de P. tomentella Wedd. subsp. incanoides y P. subtusalbida. Desde entonces, una especie adicional, $P$. rodolfo-vasquezii L. Valenzuela and I. Villalba, fue descrita para Perú (Valenzuela and Villaba 2015) dando un total de 28 especies de Polylepis.
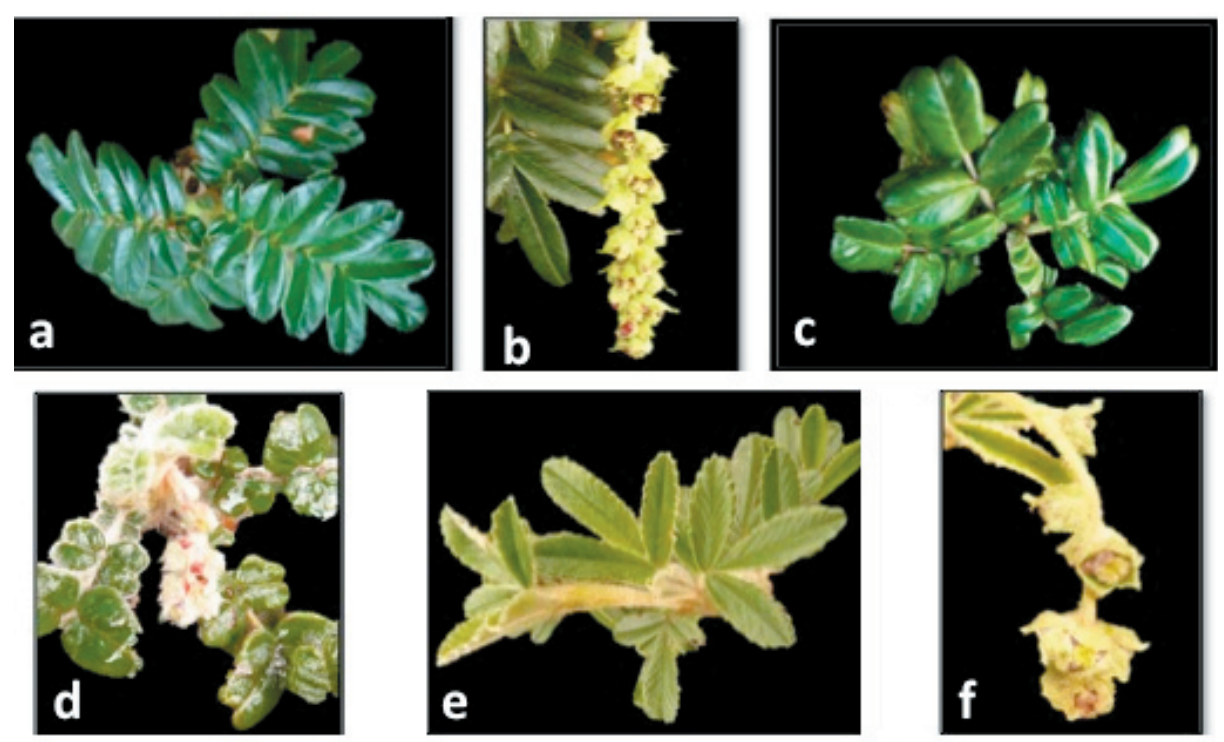

Figura 1. Grupos evolutivos utilizados en la clasificación del género Polylepis propuestos inicialmente por Simpson (1979). A) Folíolos de P. pauta. B) Inflorescencia de P. pauta (grupo sericea). C) Folíolos de P. reticulata. D) Inflorescencia de $P$. reticulata (grupo reticulata). E) Folíolos de P. racemosa. F) Inflorescencia de P. racemosa (complejo incana).

Figure 1. Evolutionary groups used in the classification of the genus Polylepis initially proposed by Simpson (1979). A) Leaflets of $P$. pauta. B) Inflorescence of $P$. pauta (sericea group). C) Leaflets of $P$. reticulata. D) Inflorescence of $P$. reticulata (reticulata group). E) Leaflets of $P$. racemosa. F) Inflorescence of $P$. racemosa (incana complex). 
Tabla 1. Problemas taxonómicos presentes en el género Polylepis basados en los tratamientos previos y en estudios locales.

Table 1. Taxonomic issues in the genus Polylepis based on previous treatments and local studies.

\begin{tabular}{|c|c|c|c|c|c|}
\hline \multicolumn{2}{|l|}{ Grupos evolutivos } & Especie & $\begin{array}{l}\text { Hibridación con } \\
\text { otras especies } \\
\text { del género }\end{array}$ & $\begin{array}{c}\text { Marcada } \\
\text { variación } \\
\text { morfológica }\end{array}$ & $\begin{array}{c}\text { Citotipos } \\
\text { (variación } \\
\text { ploidal) }\end{array}$ \\
\hline \multirow{6}{*}{\multicolumn{2}{|c|}{ Grupo sericea }} & P. canoi & & & \\
\hline & & P. lanuginosa & & & \\
\hline & & P. multijuga & & & \\
\hline & & P. pauta & $x^{1}$ & $x^{5}$ & \\
\hline & & P. pepei & & $x^{4}$ & \\
\hline & & P. sericea & $x^{1}$ & $x^{5}$ & $x^{7}$ \\
\hline \multirow{5}{*}{\multicolumn{2}{|c|}{ Grupo reticulata }} & P. hieronymi & $x^{2}$ & & \\
\hline & & P. microphylla & & & \\
\hline & & P. quadrijuga & & & \\
\hline & & P. reticulata & & $x^{1,6}$ & \\
\hline & & P. weberbaueri & & $x^{1,6}$ & \\
\hline \multirow[t]{18}{*}{ Complejo Incana } & Especies basales & P. australis & & & $x^{8}$ \\
\hline & & P. neglecta & & & \\
\hline & & $\begin{array}{l}\text { P. subsericans } \\
\text { P. rodolfo-vasquezii }\end{array}$ & & & \\
\hline & Subgrupo racemosa & P. flavipila & & $x^{4}$ & \\
\hline & & P. incarum & & $x^{2}$ & \\
\hline & & P. lanata & & $x^{2}$ & \\
\hline & & P.pacensis & $x^{3}$ & & \\
\hline & & P. racemosa & $x^{4}$ & $x^{5}$ & $\mathrm{x}^{7,9}$ \\
\hline & & P. triacontandra & $x^{2}$ & $x^{2}$ & \\
\hline & Subgrupo besseri & P. besseri & $x^{2}$ & & \\
\hline & & P. crista-galli & & & \\
\hline & & P. rugulosa & & & \\
\hline & & P. subtusalbida & $x^{2}$ & $x^{2}$ & \\
\hline & Subgrupo incana & P. incana & $x^{1}$ & $x^{1,5}$ & \\
\hline & & P. tarapacana & & & \\
\hline & & P. tomentella subsp. incanoides & & $x^{2}$ & \\
\hline & & P. tomentella subsp. nana & $x^{2}$ & $x^{2}$ & \\
\hline & & P. tomentella subsp. tomentella & $x^{2}$ & $x^{2}$ & \\
\hline
\end{tabular}

1. Romoleroux (1996); 2. Kessler (1995); 3. Kessler and Schmidt-Lebuhn (2006); 4. Segovia, C., M. Kessler, and T. Boza (observación personal); 5. Simpson (1979); 6. Mendoza and Cano (2012); 7. Segovia-Salcedo (2014);

8. Kessler et al. (2014); 9. Schmidt-Lebuhn et al. (2006).

Clasificación de las especies del género en base al conocimiento actual

A pesar de la serie de estudios sobre este género, actualmentesemantienela clasificación propuesta por Simpson en 1979, con 3 grupos evolutivos principales: sericea, reticulata y el complejo incana. Su diferenciación se centra en aspectos morfológicos y ecológicos. Los más importantes son el número de folíolos, la ubicación y el tipo de los tricomas (apéndices de la epidermis de las plantas con diferentes funciones), la longitud de las inflorescencias y la ubicación geográfica (Figura 1). Muchas de las especies pueden ser diferenciadas claramente en base a su morfología; sin embargo, debido a la existencia de ecotipos, la ubicación geográfica del individuo juega un rol fundamental en la identificación de la especie. La elevada variación morfológica intraespecífica representa un desafío para delimitar con claridad algunas especies y constituye una de las principales discrepancias en la clasificación de las especies. A continuación, se presenta una breve revisión de los grupos y la situación taxonómica de las especies que pertenecen a cada uno de ellos (Tabla 1).

Grupo sericea (6 especies). Se caracteriza por presentar hojas con numerosos pares de folíolos, inflorescencias largas con múltiples 
flores y la presencia de tricomas seríceos. Simpson (1979) lo considera como el grupo basal de este género, mientras que SchmidtLebuhn et al. (2006) lo consideraron como una agrupación no monofilética de especies basales al resto del género.

* P. canoi W. Mend. Es una especie morfológicamente diferenciable por su vaina estipular glabra, por la presencia de no más de cuatro pares de folíolos y por el hipantio del fruto, de forma romboide y con espinas pequeñas planas (Kessler and SchmidtLebuhn 2006). Es endémica de la Cordillera de Vilcabamba, Perú (Mendoza 2005). Necesita estudios más profundos para definir su situación taxonómica.

* P. lanuginosa Kunth. Se trata de una especie diferenciable en base a sus características morfológicas, centradas en una capa pequeña de tricomas lanosos bajo la capa de tricomas largos y sedosos, típicos de esta especie. Además, el ápice de sus folíolos es emarginado (Kessler and Schmidt-Lebuhn 2006). Es una especie endémica del sur del Ecuador. No tiene problemas taxonómicos.

* P. multijuga Pilg. Es una especie que se diferencia con facilidad de otras especies por el número de sus folíolos (6-8) y sus largas inflorescencias de no más de 20 flores; es endémica del norte del Perú (Kessler and Schmidt-Lebuhn 2006). No tiene problemas taxonómicos.

* P. pauta Hieron. Simpson (1979) afirmó que se podían reconocer poblaciones geográficamente separadas (e.g., en Ecuador, en el NE de Perú y en el sur de Perú hasta Bolivia). Podrían ser especies distintas. Existe una gran variación morfológica en el número y el margen de los folíolos, así como en la longitud de sus inflorescencias. Además, esta especie forma híbridos con $P$. sericea Wedd. Y con P. incana Kunth (Romoleoux 1996).

* P. pepei B. B. Simpson. Es una especie diferenciable del resto de las especies de su grupo por el tamaño pequeño de sus folíolos $(0.6-1.3 \mathrm{~cm})$ y por sus frutos con protuberancias irregulares (Kessler and Schmidt-Lebuhn 2006). Sin embargo, una descripción reciente de $P$. rodolfo-vasquezii ha demostrado la presencia de dos posibles morfoespecies: una en Perú ( $P$. rodolfo-vasquezii) y otra en Bolivia (la "verdadera" P. pepei).

* P. sericea Wedd. Esta es una de las especies más ampliamente distribuidas y de morfología más variable. Se caracteriza por poseer tricomas tomentosos y un anillo de glándulas multicelulares en el punto de unión de los foliolos (Kessler and Schmidt-Lebuhn 2006). En diferentes tratamientos taxonómicos (Benoist 1934; Macbride 1934; Cuatrecasas $1941,1942)$ se la ha considerado como otras especies ( $P$. ochracea o $P$. stueblii, entre otras). Muchas poblaciones se encuentran geográficamente aisladas y ciertos estudios sugieren que presenta diferentes citotipos, al menos en Ecuador (Segovia-Salcedo 2014; Segovia-Salcedo and Quijia 2014). Debe ser considerada como un complejo de especies. Los principales grupos geográficos se encuentran en Venezuela, en la Cordillera Occidental y Central de Colombia, en Ecuador, Cordillera Blanca, y Cuzco en Perú y Bolivia. Otro problema es Polylepis albicans, una forma morfológicamente distinta a $P$. sericea, a pesar de que Simpson (1979) las consideró como una sola especie. Su origen podría centrarse en un proceso de hibridación entre $P$. sericea y una especie del grupo reticulata.

Grupo reticulata (5 especies). El grupo se caracteriza por poseer folíolos emarginados, con tricomas panosos en el envés de las hojas.

* P. hieronymi Pilger. Es una especie morfológicamente diferenciable por sus largos tricomas seríceos en la vaina estipular (Kessler and Schmidt-Lebuhn 2006). Se reportó una hibridación con P. australis (Simpson 1978). Se distribuye en Bolivia y la Argentina.

* P. microphylla (Wedd.) Bitter. Es una especie fácilmente reconocible por el tamaño reducido de sus folíolos, en comparación con las otras especies, y por el envés y la vaina estipular con pequeños tricomas multicelulares entremezclados, de color rojo oscuro (Kessler and Schmidt-Lebuhn 2006). Es posible que se encuentre en peligro crítico por su reducido tamaño poblacional en el Ecuador. Hay poblaciones aisladas en Cuzco y Arequipa (Perú), y una sola población en Ozogoche (Ecuador), posiblemente generada por una traslocación en períodos prehispánicos (Kessler and Scchmidth-Lebuhn 2002).

* P. quadrijuga Bitter. Se trata de una especie endémica de Colombia. Se la reconoce con facilidad por la superficie rugosa en el haz de sus folíolos, por sus vainas estipulares apicalmente obtusas y por sus frutos con espinas teretes (Kessler and Schmidt-Lebuhn 2006). Sin problemas taxonómicos. 
* $P$. reticulata Hieron. Es una especie distribuida en Ecuador y en Perú. Sin embargo, las poblaciones son morfológicamente diferentes. Es posible que sean taxones diferentes. Se caracteriza por sus vainas estipulares cubiertas con tricomas seríceos, mientras que el envés de las hojas posee tricomas panosos (Kessler and SchmidtLebuhn 2006).

* P. weberbaueri Pilg. Se trata de poblaciones aisladas geográficamente, con diferencias morfológicas distribuidas en Ecuador y en Perú (Cordillera Blanca). Es probable que estudios más profundos generen dos diferentes taxones. El envés de los folíolos y las vainas estipulares presentan tricomas panosos (Kessler and Schmidt-Lebuhn 2006).

Complejo incana (17 especies, P. tomentella con 3 subespecies). Las especies poseen hojas con un número reducido de folíolos, en comparación con los otros dos grupos. Presenta inflorescencias más cortas y tricomas glandulares debajo de las hojas. El complejo incluye tres especies basales, y el resto de las especies se subdivide en tres subgrupos sobre la base de sus similitudes morfológicas.

* Especie basal P. australis Bitter. Es reconocible morfológicamente en relación con otras especies del grupo por sus vainas estipulares híspidas o glandulares, de 1 a 3 pares de folíolos y por su distribución en la Argentina (Kessler and Schmidt-Lebuhn 2006); sin embargo, su morfología es variable. Estudios recientes demuestran que existen diferentes niveles de ploidía entre las poblaciones (Kessler et al. 2014).

* Especie basal P. neglecta M. Kessler. Difiere morfológicamente de las especies de su grupo por poseer entre 2 y 4 pares de folíolos y vainas estipulares glabras o híspidas. Se distribuye principalmente en Bolivia (Kessler and Schmidt-Lebuhn 2006). Se necesitan estudios más detallados para definir su situación. No presenta conflictos taxonómicos, por el momento.

* Especie basal P. subsericans J. F. Macbr. Es reconocible morfológicamente por la presencia de folíolos más grandes en comparación con $P$. pepei, del grupo Sericea $(1.7-2.3 \mathrm{~cm})$, y de frutos con salientes irregulares aplanadas (Kessler and Schmidt-Lebuhn 2006). Su ubicación en este grupo necesita mayor análisis.

* Especie basal P. rodolfo-vasquezii L. Valenzuela and I. Villalba. Esta especie está restringida a la comunidad de Santa Rosa de Toldopamba, en el Bosque Protector de Pui-Pui, en Junín, Perú. Morfológicamente es similar a P. subsericans y P. pepei, P. rodolfovasquezii. Presenta folíolos emarginados y tricomas hirsutos, a diferencia de los folíolos crenados con tricomas erectos de $P$. subsericans (Valenzuela and Villalba 2015). Se necesitan estudios más profundos para determinar su situación taxonómica.

* Subgrupo racemosa: P. flavipila (Bitter) M. Kessler and Schmidt-Leb. Se reconoce gracias a las vainas estipulares con tricomas estrigosos y sólo un par de folíolos, raramente dos. Se distribuye al oeste del Perú (Kessler and Schmidt-Lebuhn 2006). Se necesita un conocimiento previo de su morfología. Una población cercana a Lima es morfológicamente diferente, posiblemente una nueva especie.

* Subgrupo racemosa: P. incarum (Bitter) M. Kesller and Schimidt-Leb. Es una "especie" sumamente compleja. Se le conoce únicamente en los alrededores del Lago Titicaca, donde fue plantada, pero no se conocen poblaciones naturales. Se caracteriza por sus vainas estipulares cubiertas con pelos lanosos y con 1 a 2 pares de folíolos. Tendría un origen híbrido (Kessler and Schmidt-Lebuhn 2006).

* Subgrupo racemosa: P. lanata (Kuntze) M. Kessler and Schmidt-Leb. Se trata de una especie morfológicamente diferente al resto de las especies del grupo. Tiene 2 a 3 pares de folíolos apicalmente obtusos o emarginados, en algunos casos con margen dentado ( 4 a 8 dientes por lado). El raquis de la inflorescencia está cubierto por tricomas villosos. Se ubica en Bolivia (Kessler and Schmidt-Lebuhn 2006). Es considerada una aloespecie de $P$. pacensis y $P$. besseri y se sospecha que se hibrida con el resto de las especies del grupo. Existen poblaciones en el área del Cusco tratadas como P. lanata por Mendoza y Cano (2012), pero posiblemente sean una especie distinta. Antes fueron consideradas dentro de $P$. racemosa por Simpson (1979).

* Subgrupo racemosa: P. pacensis M. Kessler and Schimdt-Leb. Se la puede diferenciar del resto de las especies del grupo sobre la base de sus características morfológicas: 2 ó 3 pares de folíolos, vainas estipulares cubiertas por ligeras espuelas, raquis de las hojas cubiertas de forma densa por pelos blancos panosos, envés de los folíolos también cubiertos densamente por pelos panosos blancos, y pelos glandulares amarillos a lo largo de las 
venas. Se localiza en Bolivia, tiene un origen híbrido y se la considera una aloespecie de $P$. lanata y $P$. triacontandra. Al parecer, se hibrida con otras especies del grupo (Kessler and Schmidt-Lebuhn 2006).

\begin{abstract}
* Subgrupo racemosa: P. racemosa Ruiz and Pav. Es una de las especies más variables y, por lo tanto, difícil desde el punto de vista taxonómico. Se caracteriza por el raquis de sus hojas cubierto por pelos villosos y por la falta de pelos glandulares amarillentos (Kessler and Schmidt-Lebuhn 2006). Es un complejo poliploide muy sembrado en proyectos de reforestación (Segovia-Salcedo 2014). No se conocen poblaciones naturales. Puede haberse generado como híbrido en tiempos prehispánicos; desde entonces fue cultivado. Originario del Perú e introducido en el Ecuador hace 40 años. En las poblaciones ecuatorianas se ha detectado variación en el número cromosómico. De igual manera, se sabe que puede hibridar con especies del mismo grupo, en especial $P$. incana (SegoviaSalcedo 2014).
\end{abstract}

* Subgrupo racemosa: P. triacontandra Bitter. Esta es una especie que se puede diferenciar de las otras especies con un conocimiento morfológico. Folíolos de forma lanceolada a ovoide, con el envés cubierto por tricomas blancos, largos, gruesos y villosos. Posiblemente tenga un origen híbrido; es una aloespecie de $P$. pacensis y $P$. lanata. Se sospecha que se hibrida con otras especies del grupo (Kessler and Schmidt-Lebuhn 2006).

* Subgrupo besseri: P. besseri Hieron. Es una especie distinguible con conocimiento de su morfología. Posee de 1 a 3 pares de folíolos con vainas estipulares sin espuelas, el raquis de la hoja cubierto densamente por pelos blancos panosos y amarillos glandulares. Se ubica en Bolivia (Kessler and Schmidt-Lebuhn 2006). Se hibrida con P. lanata y con P. subtusalbida.

* Subgrupo besseri: P. crista-galli Bitter. Es una especie diferenciable morfológicamente. Se la encuentra en el rango de distribución entre $P$. australis y $P$. neglecta. Tiene entre 1 y 2 pares de folíolos de forma ovoide y rómbica, con frutos irregularmente indentados de color verde, café o rojo. Se ubica en Bolivia y la Argentina (Kessler and Schmidt-Lebuhn 2006). Es posible que sea el resultado de una hibridación entre una especie ancestral de $P$. australis, $P$. neglecta, y de una especie del grupo besseri (Schmidt-Lebuhn et al. 2010).
* Subgrupo besseri: P. rugulosa Bitter. Esta es una especie localizada en Perú y en Chile. Algunas poblaciones, en particular en la Cordillera Occidental, presentan dificultades para su diferenciación en base a detalles morfológicos. La superficie de los folíolos es muy rugosa. Posee hojas e inflorescencias sin pelos glandulares amarillos (Kessler and Schmidt-Lebuhn 2006), pero sí cubiertas por pelos blancos panosos.

* Subgrupo besseri: P. subtusalbida (Bitter) M. Kessler and Schimdt-Leb. Se caracteriza por folíolos largos, elípticos, lanceolados. Se ubica en Bolivia (Kessler and Schmidt-Lebuhn 2006). Se encontraron putativos híbridos con $P$. lanata y $P$. besseri que necesitan análisis más profundos.

* Subgrupo besseri: P. tomentella Wedd. subsp. incanoides M. Kessler. La especie tiene folíolos con márgenes dentados, con los ápices obtusos a poco agudos. El envés de los folíolos está cubierto, o no, por pelos glandulares amarillos. La parte superior de los folíolos es glabra o poco pilosa (Kessler and Schmidt-Lebuhn 2006). Está distribuido en Perú y Bolivia. Existen algunas poblaciones morfológicamente diferentes de esta especie en el Perú; fueron catalogadas por Mendoza y Cano (2012) como P. subtusalbida, pero pueden ser una especie distinta.

* Subgrupo incana: $P$. incana Kunth. Es una especie compleja en el aspecto taxonómico, que se caracteriza por tener la parte superior de los folíolos sin resina, glabros, con margen crenado y ápices emarginados. Diferentes poblaciones (Ecuador, Cordillera Blanca, Cusco) están separadas geográficamente, tienen diferentes características ecológicas y son morfológicamente distintas. Pueden ser diferentes especies. Además, $P$. incana es una especie que fue muy usada en programas de reforestación, lo que complicó su situación taxonómica. Tiene la capacidad de hibridar con las especies de su grupo y de los dos otros grupos (P. sericea y P. pauta) (Romoleorux 1996).

* Subgrupo incana: P. tarapacana Phil. Se trata de una especie en la que la superficie de los folíolos es glabra y está cubierta por una resina amarillenta. El margen de los folíolos es entero o medianamente crenado. Se distribuyen en Perú, Chile y Bolivia. Es distinta morfológicamente a las otras especies del grupo, especialmente en poblaciones aisladas. 
* Subgrupo incana: P. tomentella Wedd. subsp. nana M. Kessler. Se puede distinguir a esta especie de otras similares por la falta de pelos glandulares, y es una versión enana (alcanza hasta $1.5 \mathrm{~m}$ ) de $P$. tomentella. Se la conoce en una sola ubicación, en la provincia de Arani, Bolivia. Se necesitan más análisis para determinar si debe ser considerada como otra especie (Kessler and Schmidt-Lebuhn 2006).

* Subgrupo incana: P. tomentella Wedd. subsp. tomentella. Es una especie morfológicamente distinta, ubicada desde el sur del Perú hasta el noroeste de la Argentina. Se observaron individuos híbridos a nivel morfológico con $P$. tarapacana. La población de Perú es morfológicamente diferente a la población de la Argentina, por lo que se necesitan más estudios.

* Posible nueva especie: $P$. pallidistigma Bitter. No se le ha tomado en cuenta en los últimos tratamientos (Kessler and SchmidthLebuhn 2005; Mendoza and Cano 2011). Las poblaciones en Perú, en la cadena montañosa del Lago Titicaca, con características morfológicas similares a $P$. besseri de acuerdo con Simpson (1979), podrían pertenecer a esta especie. Con estudios más profundos se la podría re-instaurar.

\section{Discusión}

\section{Desafíos y vacíos de información para la delimitación de especies}

Los problemas asociados con la identificación y la separación de las especies de Polylepis requieren un mejor entendimiento de los procesos biológicos que influyen a diferentes escalas, tanto a nivelinter-comointraespecífico. Si bien existen varias revisiones sobre la taxonomía del género, todavía hay vacíos sustanciales de información sobre la biología de las especies. Esto dificulta la posibilidad de establecer criterios para delimitar de forma adecuada a las especies. En esta sección realizamos un análisis sobre los mencionados vacíos de información e identificamos los campos de estudio para poder contribuir a un mejor entendimiento de los procesos que influyen en la delimitación de las especies.

Filogenia. Schmidt-Lebuhn et al. (2006a) construyeron una filogenia de Polylepis en base a AFLP y a caracteres morfológicos. Esta filogenia apoya las hipótesis de evolución y de diversificación del género (Bitter 1911; Simpson 1979, 1986; Kessler 1995). Es decir, una tendencia a la disminución en el número de hojas y de flores, y el tamaño de las plantas asociado con el incremento de la altitud. Sin embargo, la señal filogenética todavía no está totalmente resuelta debido a un ruido posiblemente asociado con introgresión e hibridación. Este es el caso de P. subtusalbida, que presenta individuos morfológicamente similares a $P$. besseri, lo que sugiere una introgresión híbrida (Kessler and SchmidtLebuhn 2006). Además, esta filogenia no incluye tres especies recientemente descritas (P. pacensis, P. canoi y P. rodolfo-vasquezii).

La genómica provee nuevas oportunidades para mejorar la filogenia de Polylepis. El campo de filogenómica avanzó rápidamente durante la última década debido a la disponibilidad amplia de técnicas de bajo costo como la secuenciación de nueva generación (nextgeneration sequencing), así como gracias a nuevas herramientas informáticas que permiten adquirir y procesar esta información (Barrett et al. 2016). La filogenómica permite reconstruir las relaciones evolutivas entre especies a través de la comparación de genomas enteros o parciales; ya demostró ser útil con grupos de especies producto de una radiación adaptativa, como es el caso del clado del tomate silvestre Lycopersicon (Pease et al. 2016).

Niveles de ploidía. Schmidt-Lebuhn et al. (2010) demostraron por primera vez la existencia de poliploidía en el género. Además, los autores infirieron los niveles de ploidía y mostraron una clara progresión de diploidía en las especies que habitan los bosques montanos (e.g., P. pepei, P. microphylla y $P$. quadrijuga), hacia tetraploidía ( $P$. australis y $P$. subsericans) y hacia la octoploidía (P. racemosa) en las especies que habitan ecosistemas a mayores elevaciones, particularmente en el grupo incana. Adicionalmente, el estudio mostró casos de ploidía mixta en el género, en particular en $P$. australis y $P$. pauta, que presentan individuos poli- y diploides. Kessler et al. (2014) estudiaron en detalle los niveles de ploidía de $P$. australis y hallaron una variabilidad intraespecífica notable y patrones geográficos no fácilmente interpretables. Estos estudios sugieren que la poliploidización puede ser producto de la hibridación. Sin embargo, todavía queda por estudiar si esta variación es producto de diferencias geográficas, ecológicas o morfológicas.

Estudios genéticos. Los estudios genéticos de poblaciones son clave para identificar el 
flujo genético entre las especies de Polylepis. Sin embargo, hasta la fecha, no existen estudios publicados que hayan evaluado tasas de flujo genético entre especies, incluso en aquellas en las que existen evidencias de hibridación. Por fortuna, existen estudios de estructura poblacional utilizando marcadores genéticos (AFLP; amplified fragment length polymorphism) en varias especies de Polylepis. Estos estudios proveen información fundamental acerca del flujo genético entre poblaciones y permiten entender variaciones pasadas en la distribución de las especies, así como el papel del cambio climático y las actividades antrópicas. Por ejemplo, $P$. australis y $P$. tarapacana muestran bajos niveles de diferenciación genética entre poblaciones, lo cual sugiere un extenso flujo genético que conecta poblaciones a lo largo del rango altitudinal de su distribución (Hensen et al. 2015; Peng et al. 2016), con excepción de las poblaciones de $P$. australis distribuidas al norte de la Argentina, que presentan una diferenciación genética elevada (Hensen et al. 2011). En contraste, P. incana posee una diversidad genética mayor dentro de las poblaciones que entre las poblaciones, lo que sugiere un aislamiento espacial fuerte (Hensen et al. 2012).

Diferenciación morfológica. La identificación de las especies de Polylepis se basa mayormente en caracteres morfológicos. Sin embargo, la morfología de algunas especies del género es polimórfica y diferentes individuos de una misma especie pueden presentar una variación morfológica amplia. Por ejemplo, $P$. racemosa muestra una gran variación en varios caracteres (e.g., densidad y tipo de indumento de los folíolos, posición de las inflorescencias y densidad de pelos en los estambres); incluso, algunos individuos son morfológicamente más similares a P. besseri (Kessler and SchmidtLebuhn 2006). La variación morfológica intraespecífica podría deberse a hibridación entre poblaciones cercanas, a plasticidad fenotípica o a adaptaciones locales (Kessler and Schmidt-Lebuhn 2006). Alternativamente, otras especies poseen una morfología muy similar. Podrían estar siendo agrupadas dentro de la misma especie por errores de identificación, aunque representen entidades taxonómicas separadas.

Las numerosas revisiones taxonómicas del género (Bitter 1911; Simpson 1979; Kessler 1995; Kessler and Schmidt-Lebuhn 2006) resaltan la variación morfológica elevada que existe en Polylepis, así como la dificultad para su identificación. Por esta razón, es fundamental considerar que la identificación taxonómica debe incluir varios individuos de una misma población y, preferentemente, material de ramas jóvenes y maduras para asegurar que los folíolos y las estípulas mantengan los pelos (Kessler and SchmidtLebuhn 2006). Además, los híbridos pueden ser frecuentes, por lo que para identificarlos se necesitan claves taxonómicas y una extensa colección de referencia.

Estudios ecológicos. La plasticidad fenotípica intraespecífica es uno de los principales factores que afectan la delimitación de las especies (Kessler 1995; Kessler and SchmidtLebuhn 2006). Sin embargo, hay muy pocos estudios ecológicos que hayan evaluado si la variación morfológica observada está asociada con variaciones ambientales o es producto de adaptaciones genéticas. Toivonen et al. (2014) llevaron a cabo un estudio para identificar el grado de plasticidad de rasgos funcionales morfológicos y fisiológicos, y el rol del determinismo genético en 9 especies de Polylepis. Los resultados mostraron que algunos caracteres funcionales están determinados genéticamente y proveen una capacidad adaptativa a las especies. Por ejemplo, el área de las hojas y la tasa de fotosíntesis máxima aumentan con la temperatura. Sin embargo, otros caracteres mostraron una señal filogenética débil, lo que sugiere que gran parte de la variación no se puede atribuir sólo a la filogenia, sino a una especialización compleja de las especies a la variación ambiental.

Los experimentos de trasplantes recíprocos (en inglés, common garden) pueden ayudar a determinar si la variabilidad fenotípica observada tiene una base genética o es el resultado de la plasticidad fenotípica en Polylepis. Los estudios de trasplantes consisten en hacer crecer individuos de diferentes poblaciones en un ambiente común para poder estudiar las bases de caracteres complejos, incluyendo morfológicos y fisiológicos. Esta herramienta demostró ser útil que permite quitar los efectos que podrían surgir por variabilidad ambiental, en especial cuando existen gradientes como la elevación (Ebeling et al. 2011). Sin embargo, la gran limitación para implementar estos experimentos con Polylepis es la longevidad de las especies, puesto que se hace difícil mantener los individuos el tiempo suficiente como para que puedan producir descendencia. En este caso, el jardín botánico de la Universidad Mayor 
de San Andrés, en Bolivia, constituye una excelente oportunidad para llevar a cabo este tipo de estudios, puesto que posee individuos de varias especies de Polylepis provenientes de diferentes lugares de Bolivia, en algunos casos cultivadas desde 1991.

Biología reproductiva. La biología reproductiva de Polylepis es actualmente uno de los aspectos menos estudiados del género. Una de las limitantes existentes es la falta de información sobre la fenología reproductiva y los sistemas de incompatibilidad. Hasta la fecha, sólo existen dos estudios publicados sobre la biología reproductiva de $P$. besseri (Hensen 1994) y P. tomentella (Domic etal. 2013), que muestran patrones fenológicos similares, incluyendo la floración durante la época seca. El sistema de incompatibilidad sólo fue caracterizado para $P$. australis y $P$. tomentella. Las dos especies son autocompatibles y poseen la capacidad de producir frutos y semillas por autopolinización y polinización cruzada (Seltmann et al. 2009a; Domic et al. 2017).

Repetir estos estudios en otras especies de Polylepis contribuiría a entender el grado de aislamiento reproductivo, las barreras de aislamiento prezigóticas y el potencial de hibridación entre las especies. Realizar experimentos in situ de polinización cruzada para las especies que poseen una distribución simpátrica (e.g., P. racemosa y P. besseri) y que muestran evidencias de hibridación permitiría identificar la capacidad de las especies para producir semillas viables por polinización cruzada.

\section{Implicancias para la conservación y la restauración}

La taxonomía es una herramienta fundamental para entender la biodiversidad y su organización, ya que nos permite descubrir, reconocer y clasificar taxa. Esta información brinda la posibilidad de describir tendencias y estatus de especies, riesgos de extinción, datos de inventarios que directamente pueden influir en decisiones de conservación (McNeely 2002; Mace 2014; Tsang et al. 2016). De ahí que la conservación efectiva de especies requiera la definición de una unidad taxonómica que refleje la realidad biológica del organismo y que pueda ser documentada en el tiempo y en espacio (Tsang et al. 2016). Estos parámetros no siempre son fáciles de identificar, como en el caso de algunas especies de Polylepis que todavía somos incapaces de diferenciar y clasificar por falta de información taxonómica. En algunos casos, el problema se centra en el concepto de especie. Existen varios conceptos de especie; sin embargo, lo importante es que los taxónomos sean explícitos en cuál concepto emplean para sus estudios. Esto evitaría confusiones en las áreas afines (Townsend 2006).

Sin una taxonomía estructurada del género Polylepis no podemos asegurar ni que los estudios experimentales sean repetibles ni que la definición de áreas de conservación y manejo sean las más adecuadas. Por esta razón, antes de iniciar un estudio ecológico, de manejo o de conservación es fundamental realizar una revisión taxonómica básica sobre el organismo que estamos trabajando, con una visión regional y no local, para evitar perpetuar errores. Otro aspecto importante es la filogenia de este género andino, todavía no resuelta debido a potenciales procesos de hibridización, poliplodía y rápida radiación (Segovia-Salcedo 2014). Estos datos son vitales para reconstruir el pasado. Las filogenias proveen una línea base para examinar hipótesis evolutivas y biogeográficas, algunas de las que motivarán decisiones correctas en conservación. Las filogenias pueden ayudar a predecir puntos calientes de biodiversidad, a definir cuán diferentes son las poblaciones entre sí, así como a identificar linajes únicos que son críticos para la conservación genética de una especie (Tsang et al. 2016). Al no tener esta información en Polylepis, algunas iniciativas de conservación de estos bosques andinos podrían no estar cumpliendo los objetivos planteados.

Estudios taxonómicos recientes de Polylepis (Kessler and Schmidth-Lebuhn 2005; Mendoza and Cano 2011) demostraron que existen diferencias locales, probablemente por el proceso de diversificación durantela formación de los Andes y de las cadenas montañosas aledañas. Durante, la formación de las montañas se generan diferentes elevaciones, exponen una variedad sustratos, climas, y tipos de suelo. Estos procesos producen una serie de microhábitats donde las especies pueden adaptarse a los cambios locales, especialmente en un escenario como los Andes, donde los hábitats están geográficamente aislados (Xing and Ree 2017). De ahí, la importancia de estudios más amplios y detallados de cada una de las especies de Polylepis en sus rangos de distribución geográfica para entender cómo cada uno de estos parámetros están actuando en los procesos de especiación. De igual manera, al iniciar estudios en una especie 
se deben analizar, en la medida de lo posible, datos de todo el rango de distribución para generar objetivos específicos que aporten a una visión regional. Definitivamente, nuevos estudios pueden clarificar el estatus de poblaciones aisladas, y el reconocimiento de estas especies pueden permitir adecuadas estrategias de conservación y manejo, como es el caso de P. pallidistigma.

Información taxonómica y sistemática es esencial para aspectos cruciales de la conservación de los bosques de Polylepis. El correcto estatus taxonómico de una especie es utilizado por autoridades locales e internacionales en la protección de la vida silvestre, como la Convención sobre el Comercio Internacional de Especies Amenazadas de Fauna y Flora Silvestres (CITES), la convención de los humedales (RAMSAR) y la lista roja de especies de la Unión Internacional para la Conservación de la Naturaleza (UICN). En esta última, 14 especies de Polylepis están consideradas como vulnerables y una de ellas ( $P$. tarapacana), como en bajo riesgo, ya que cumplen los parámetros de reducción dehábitat $(\mathrm{A})$, reducción continua (B) y hábitat severamente fragmentado (UICN 2017; Tejedor-Garavito Newton and Oldfield 2015). Las reglamentaciones a nivel local o regional se basan en listas de especies y en las definiciones de especie amenazada o en peligro (Tsang et al. 2016; McNeely 2002). En algunas ocasiones, estas definiciones pueden generar acciones de conservación; como prerequisito, las especies deben estar en listas nacionales o internacionales. Las proporciones de especies amenazadas pueden ser calculadas por país o por área, y esta información es utilizada para determinar prioridades para destinar fondos de conservación a nivel local o regional (Mace 2004).

La protección a nivel nacional puede variar en cada uno de los países, pero en la mayoría de ellos, la unidad de conservación es la especie, que en el caso de Polylepis no está claramente definido para algunos individuos por su taxonomía compleja. Esto genera un desafío para los tomadores de decisiones, quienes en el caso del género Polylepis deberían centrar sus lineamientos en la mayor cantidad de información existente.

En estudios ecológicos y de restauración es crítico definir con certeza la especie e incluso la población evolutivamente más cercana para evitar procesos de hibridización y pérdida de linajes evolutivos que estén mejor adaptados a las condiciones locales y, al mismo tiempo, asegurar el éxito del proceso. Muchos procesos de translocación de especies sin un respaldo taxonómico han complicado la situación de delimitación de las especies de este género. Esto adquiere importancia en especies de Polylepis con una amplia distribución como el caso de $P$. incana, $P$. racemosa y $P$. sericea, entre otras.

El punto central es proveer de una estructura dentro de los estudios, para que los datos obtenidos en el campo sean un aporte en los procesos de conservación de estos bosques. Para esto, taxónomos y conservacionistas debemos trabajar de manera conjunta para delimitar las unidades de conservación, para evitar ambigüedades y para generar estrategias claras (Garnett and Christidis 2017; Townsend 2006; Mace 2004), ya que la implementación de estrategias de conservación es la actividad más importante que debemos realizar como comunidad.

\section{Conclusiones}

El género Polylepis posee 28 especies localizadas en los ecosistemas altoandinos. A pesar de las varias revisiones taxonómicas del género, todavía se dan algunas incertidumbres. Si bien existen estudios sobre el género, en comparación con otras especies de flora andina, la delimitación de las especies todavía constituye un desafío para los taxónomos y sistemáticos. Los marcadores moleculares constituyen una herramienta poderosa que podría ayudar de manera significativa a mejorar la delimitación de las especies y a aportar con la caracterización de la variación intraespecífica. Consideramos importante realizar más estudios sobre la morfología, la biología reproductiva y la ecología de las especies para ayudar a entender los procesos involucrados en la diversificación. Una de las perspectivas que necesitan ser analizadas es el concepto de especie que se utiliza en cada uno de los estudios taxonómicos del género, lo que puede estar creando un mayor número de especies. Se debe contemplar la adopción de un concepto de especie que permita unificar los datos morfológicos, reproductivos, biogeográficos, genéticos y moleculares $\mathrm{y}$, de esa manera, identificar linajes que hayan evolucionado de manera independiente. Mientras tanto, resulta fundamental trabajar de forma interdisciplinaria entre taxónomos, sistemáticos y ecólogos para basar nuestras 
investigaciones y proyectos en la información taxonómica más actualizada. Esta revisión provee el marco necesario para identificar líneas de investigación $\mathrm{y}$, de esa manera, contribuir a la delimitación de especies. Al mismo tiempo, ayuda a compartir información sobre la compleja realidad taxonómica de este género, con el objetivo de que sea incorporada a proyectos de reforestación y de restauración, con el fin último de contribuir a conservar estos bosques altamente amenazados.

Agradecimientos. Los autores quieren agradecer a los organizadores del V Congreso Internacional de Ecología y Conservación de Bosques de Polylepis.

\section{RefERENCIAS}

Barrett, C. F., C. D. Bacon, A. Antonelli, A. Cano, and T. Hofmann. 2016. An introduction to plant phylogenomics with a focus on palms. Botanic Journal of the Linnean Society 182:234-255.

Benoist, R. 1934. Descripton d' especes nouvelles de phanérogames de l’Equateur. Bulletin de la Société Botanique de France 81:324-326.

Bitter, G. 1911. Revision der Gattung Polylepis. Botanische Jahrbücher für Systematik, Pflanzengeschichte und Pflanzengeographie 45:564-656.

Bitter, G. 1913. UberverschiedeneVarietaten der Polylepis australis. Reptertorium Specierum Novarum Regni Vegetabilis 12:477-479.

Chung, K. -S., W. Elisens, and J. Skvarla. 2010. Pollen Morphology and its phylogenetic significance in the tribe Sanguisorbeae (Rosaceae). Plant Systematics and Evolution 285:135-148.

Cuatrecasas, J. 1941. Notas a la flora de Colombia, IV. Revista de la Academica Colombiana de Ciencias Exactas, Físicas y Naturales 4:337-348.

Cuatrecasas, J. 1942. Notas a la flora de Colombia, V. Revista de la Academia Colombiana de Ciencias Exactas, Físicas y Naturales 5:16-39.

De Queiroz, K., and J. Gauthier. 1992. Phylogenetic taxonomy. Annual Review of Ecology and Systematics 23:449480.

Domic, A. I., E. Mamani, and G. Camilo. 2013. Fenología reproductiva de la kewiña (Polylepis tomentella, Rosaceae) en la puna semihúmeda de Chuquisaca, Bolivia. Ecología en Bolivia 48:31-45.

Domic, A. I., P. Bernhardt, R. Edens-Meier, G. R. Camilo, and J. M. Capriles. 2017. Pollination Ecology of Polylepis tomentella (Rosaceae), an Andean Anemophilous Tree Presenting a Potential Flower-Fungal Infection. International Journal of Plant Sciences 178(7):512-521.

Ebeling, S. K., J. Stöcklin, I. Hensen, and H. Auge. 2011. Multiple common garden experiments suggest lack of local adaptation in an invasive ornamental plant. Journal of Plant Ecology 4(4):209-220.

Eriksson, T., M. Hibbss, A. Yoder, C. Delwiche, and M. Donoghue. 2003. The phylogeny of Rosoideae (Rosaceae) based on sequences of the internal transcribed spacers (ITS) of nuclear ribosomal DNA and the trnL/F region of chloroplast DNA. International Journal of Plant Sciences 164:197-211.

Evans, R., and C. Campbell. 2002. The origin of the apple subfamily (Maloideae; Rosaceae) is clarified by DNA sequence data from duplicated GBSSI genes. American Journal of Botany 89:1478-1484.

Evans, R., L. Alice, C. Campbell, E. Kellog, and T. Dickinson. 2000. The Granule-Bound Starch Synthetase (GBSSI) Gene in the Rosaceae: Multiple Loci and Phylogenetic Utility. Molecular Phylogenetic and Evolution 17:388-400.

Fjedlså, J., and M. Kessler. 1996. Conserving the biological diversity of Polylepis woodlands of the highlands of Peru and Bolivia. A contribution to sustainable natural resource management in the Andes. NORDECO, Copenhagen.

Garnett, T. S., and L. Christidis. 2017. Taxonomy Arachy hampers conservation. Science 547:29-27

Helfgott, D. M., J. Francisco-Ortega, A. Santos-Guerra, R. K. Jansen, and B. B. Simpson. 2000. Biogeography and breeding system evolution of the woody Bencomia alliance (Rosaceae) in Macaronesia Based on ITS Sequence Data. Systematic Botany 25:82-97.

Hensen, I. 1995. Estudios ecológicos y fenológicos sobre Polylepis besseri Hieron en la Cordillera Oriental boliviana. Ecología en Bolivia 23:21-32.

Hensen, I., A. Cierjacks, H. Hirsch, M. Kessler, K. Romoleroux, D. Renison, and K. Wesche. 2012. Historic and recent fragmentation coupled with altitude affect the genetic population structure of one of the world's highest tropical tree line species. Global Ecology and Biogeography 21:455-464.

Hensen, I., I. Teich, H. Hirsch, H. von Wehrden, and D. Renison. 2011. Range-wide genetic structure and diversity of the endemic tree line species Polylepis australis (Rosaceae) in Argentina. American Journal of Botany 98:1825-1833.

Hieronymus, G. Plantae. Lehmannianae Botanische Jahrbücher für Systematik, Pflanzengeschichte und Pflanzengeographie 20(III supplement 49):1-72.

Hutchinson, J. 1964. The genera of flowering plant. Oxford University Press, Oxford.

IUCN. 2017. The IUCN Red List of Threatened Species. Version 2017-2. URL: www.iucnredlist.org.

Kerr, M. 2003. A phylogenetic and biogeographic analysis of Sanguisorbeae (Rosaceae) with emphasis of the Pleistocene radiation of the high Andean genus Polylepis. PhD thesis. University of Maryland, Maryland. USA.

Kessler, M. 1995. The genus Polylepis (Rosaceae) in Bolivia. Candollea 50:131-171. 
Kessler, M. 2002. The "Polylepis Problem": Where do we stand? Ecotropica 8:97-110.

Kessler, M., A. Kühn, V. G. Solís Neffa, and I. Hensen. 2014. Complex geographical distribution of ploidy levels in Polylepis australis (Rosaceae), an endemic tree line species in Argentina. International Journal of Plant Sciences 175: 955-961.

Kessler, M., and A. N. Schmidt-Lebuhn. 2006. Taxonomical and distributional notes on Polylepis (Rosaceae). Organisms, Diversity and Evolution 6:67-69.

Kuntze, C. E. O. 1898. Revisio generum plantarum. Volume 3, CCCXX. Leipzig. Pp. 576.

Macbride, J. F. 1934. New or renamed spermatophytes mostly Peruvian. Candollea 5:346-402.

Mace, M. G. 2004. The role of taxonomy in species conservation. Phil. Trans R Soc Lond B 359:711-719.

McNeely, J. A. 2002. The role of taxonomy in conserving biodiversity. Journal for Nature Conservation 10:145-153.

Mendoza, W. 2005. Especie nueva de Polylepis (Rosaceae) de la cordillera Vilcabamba (Cusco, Perú). Revista Peruana de Biología 12(1):103-106.

Mendoza, W., and A. Cano. 2012. El género Polylepis en el Perú: taxonomía, morfología y distribución. Editorial Académica Española. España. Pp. 120.

Morgan, D., D. Soltis, and K. Robertson. 1994. Systematic and evolutionary implications of rbcL sequence variation in Rosaceae. American Journal of Botany 81:890-903.

Pease J. B., D. C. Haak, M. W. Hahn, and L. C. Moyle. 2016. Phylogenomics reveals three sources of adaptive variation during a rapid radiation. PLOS Biology 14:e1002379.

Peng, Y., S. Lachmuth, S. C. Gallegos, M. Kessler, P. M. Ramsay, D. Renison, R. Suárez, and I. Hensen. 2015. Pleistocene climatic oscillations rather than recent human disturbance influence genetic diversity in one of the world's highest treeline species. American Journal of Botany 102:1676-1684.

Pérez de la Paz, J. 2004. Rosaceae-Sanguisorbeae de Macaronesia: género Macetella, Bencomia y Dendropoterium. Palinología, biogeografía, sistemas sexuales y filogenia. Botanica Macaronesica 25:95-126.

Pilger, R. 1906. Rosaceae andinae. Botanische Jahrbücher für Systematik, Pflanzengeschichte und Pflanzengeographie 37:534-541.

Potter, D., F. Gao, P. Bortiri, S. Oh, and S. Baggett. 2002. Phylogenetic relationships in Rosaceae inferred from chloroplast matK and trnL-trnF nucleotide sequence data. Plant Systematics and Evolution 231:77-89.

Potter, D., T. Ericsson, R. Evans, S. Oh, J. Smedmark, D. Morgan, M. Kerr, K. R. Robertson, M. Arsenault, T. A. Dickinson, and C. S. Campbell. 2007. Phylogeny and classification of Rosaceae. Plant Systematics and Evolution 266:5-43.

Romoleroux, K. 1996. Flora of Ecuador: 79. Rosaceae. Publishing House of the Swedish Research Council, Stockholm.

Schmidt-Lebuhn, A. N., J. Fuchs, D. Hertel, H. Hirsch, J. Toivonen, and M. Kessler. 2010. An Andean radiation: polyploidy in the tree genus Polylepis (Rosaceae, Sanguisorbeae). Plant Biology 12:917-926.

Schmidt-Lebuhn, A. N., M. Kessler, and M. Kumar. 2006. Promiscuity in the Andes: Species relationships in Polylepis (Rosaceae, Sanguisorbeae) based on AFLP and morphology. Systematic Botany 31:547-559.

Segovia-Salcedo, M. C., and P. Quijia-Lamina. 2014. Citogeografía de cuatro especies de Polylepis (Rosacea) en el Ecuador: Información relevante para el manejo y conservación de los bosques andinos. En F. Cuesta, J. Sevink, L. D. Llambi, B. De Bievre and J. Posner (eds.). Avances en investigación para la conservación de los páramos andinos, CONDESAN.

Segovia-Salcedo, M. C. 2014. New insights into the evolutionary history of the complex Andean genus Polylepis (Rosaceae: Sanguisorbeae) and implications for conservation and management. PhD thesis. University of Florida. USA. Pp. 182.

Seltmann, P., A. Cocucci, D. Renison, A. Cierjacks, and I. Hensen. 2009a. Mating system, outcrossing distance effects and pollen availability in the wind-pollinated treeline species Polylepis australis BITT. (Rosaceae). Basic and Applied Ecology 10:52-60.

Seltmann, P., D. Renison, A. Cocucci, I. Hensen, and K. Jung. 2007. Fragment size, pollination efficiency and reproductive success in natural populations of wind-pollinated Polylepis australis (Rosaceae) trees. Flora 202:547-554.

Seltmann, P., I. Hensen, D. Reninson, K. Wesche, S. Ploch, J. Duenas, A. Cocucci, and K. Jung. 2009b. Biparental inbreeding depression, genetic relatedness and progeny vigour in a wind-pollinated treeline species in Argentina. Plant Ecology 205:155-164.

Simpson, B. B. 1979. A revision of the genus Polylepis (Rosaceae: Sanguisorbeae). Smithsonian Contributions to Botany 43:1-62.

Simpson, B. B. 1986. Speciation and specialization of Polylepis in the Andes. Pp. 304-316 en F. Vuilleumier and M. Monasterio (eds.). High altitude tropical biogeography. Oxford University Press, Oxford.

Tejedor-Garavito, N., A. Newton, and S. Oldfield. 2015. Regional Red List Assessment of tree species in upper montane forests of the Tropical Andes. Oryx doi:10.1017/S0030605315000198.

Toivonen, J. M., V. Horna, M., Kessler, K., Ruokolainen, and D., Hertel. 2014. Interspecific variation in functional traits in relation to species climatic niche optima in Andean Polylepis (Rosaceae) tree species: evidence for climatic adaptations. Functional Ecology 41:301-312.

Townsend-Peterson, A. 2008. Taxonomy is important in conservation: a preliminary reassessment for Philippine species-level bird taxonomy. Bird Conservation International 16:155-173.

Tsang, S. M., A. L. Cirranello, N. B. Simmon, P. J. J. Bates, and N. B. Simmons. 2016. The Roles of Taxonomy and 
Systematics in Bat Conservation. Pp. 503-538 en C. Voigt and T. Kingston (eds.). Bats in the Anthropocene: Conservation of Bats in a Changing World. Springer Open. Lubbock, Texas.

UNEP-WCMC. 2004. United Nations Environment Programme. World Conservation Monitoring Center. URL: www.unep-wcmc.org.

Valenzuela, G., and I. Villalba. 2015. A new species of Polylepis from Perú. Arnaldoa 22(2):329-338.

Weimarck, H. 1934. Monograph of the genus Cliffortia. Universitetsbokhandeln, Lund.

Xing, Y., and R. Ree. 2017. Uplift-driven diversification in the Hengduan Mountains, a temperate biodiversity hotspot. PNAS 114(17):E3444-E3451 doi: 10.1073/pnas.1616063114. 